\title{
Effects of exercise training on the hemodynamics in patients with chronic heart failure
}

\author{
Shinji Satoh*, Miyuki Kamo, Tomoko Ohtsuka and Koji Hiyamuta \\ Division of Cardiology and Clinical Research Institute, National Hospital Organization, Kyushu Medical Center, Japan
}

\begin{abstract}
Background and purpose: It is still unclear physiological mechanisms of preserved exercise-tolerance in patients with stable chronic heart failure, irrespective decreased left ventricular ejection fraction. We tried to investigate underlying mechanisms.

Methods: Consecutive 62 patients with stable chronic heart failure under oral medications were enrolled (NYHA II-III). Exercise test was performed on a symptomlimited bicycle ergometer. We analyzed changes in cardiac parameters by means of non-invasive whole body electrical bio-impedance using NICaS apparatus during exercise. Age (yrs, 25\%-75\% range): 63 (49-72). Ejection Fraction (\%): 61 (38- 69). Basic diseases of heart failure: Idiopathic dilated cardiomyopathy 22; Valvular heart disease 22; Old myocardial infarction 15; Congenital heart disease 2; Hypertensive heart disease 1.
\end{abstract}

Results: Comparing before and after exercise, heart rate was significantly increased after exercise, but there were no significant changes in mean blood pressure. While total peripheral vascular resistance index tended to be decreased, cardiac index tended to be increased.

Conclusions: There might exist beneficial physiological mechanisms to decrease total peripheral vascular resistance accompanied with increasing cardiac output during exercise in patients with stable chronic heart failure associated with preserved exercise-tolerance. Exercise-induced peripheral vasodilation may underlie

\section{Introduction}

Exercise training has been established as an important treatment strategy in patients with chronic heart failure [1]. It improves the functional and aerobic capacity, delays the anaerobic metabolism [2], and improves the autonomic balance, which was associated with lower rates of mortality [3] and hospital readmission for heart failure during a long-term follow-up [4]. Although improvements in the endothelial function, which is impaired in patients with heart failure [5], has been proposed as one of the underlying mechanisms [6], the physiological mechanisms underlying the preserved exercise tolerance in patients with stable chronic heart failure [7], irrespective of a decreased ejection fraction, are still unclear. We herein investigated these underlying mechanisms.

\section{Methods}

This study was a prospective observational study of 62 patients with stable chronic heart failure who were well controlled with oral medication (NYHA II-III). Exercise training was performed on a symptom-limited bicycle ergometer (Fukuda Denshi, Tokyo, Japan) according to the current guidelines [8].

Patients with autoimmune or inflammatory diseases (including those treated with steroids and/or non-steroidal anti-inflammatory drugs), active infections, malignant diseases or liver cirrhosis were excluded from the study.

All patients who underwent Percutaneous Coronary Intervention (PCI) were administered pharmacological treatment before including the administration of aspirin (100mg), heparin (5,000IU) and clopidogrel (a loading dose of $300 \mathrm{mg}$ ). The standard therapy after PCI included aspirin, clopidogrel, beta-blockers, lipid-lowering agents, such as statins, and angiotensin-converting enzyme inhibitors or angiotensin II receptor blockers, according to the current guidelines [9]. Patients with heart failure were administered standard pharmacological treatments, including angiotensin-converting enzyme inhibitors or angiotensin II receptor blockers, beta-blockers and aldosterone antagonists, according to the current guidelines [8].

We measured the cardiac parameters, such as the heart rate, mean blood pressure, total peripheral vascular resistance and cardiac output by means of non-invasive whole body electrical bio-impedance using an $\mathrm{NICaS}^{\mathrm{TM}}$ apparatus (NICAS 2204 Slim, NImedical, KfarMalal, Israel), and compared the changes in these parameters before and after exercise.

Blood tests were performed on admission under either fasting or casual conditions.

Written informed consent for cardiac catheterization and data utilization was obtained from each patient, and the study protocol conformed to the ethical guidelines of the 1975 Declaration of Helsinki, as reflected in a priori approval from our institution's human research committee.

Correspondence to: Shinji Satoh, Division of Cardiology and Clinical Research Institute, National Hospital Organization Kyushu Medical Center, 1-8-1 Jigyohama, Chuo-ku, Fukuoka 810-8563, Japan, Tel: +81-92-852-0700; Fax: +81-92-847-8802; E-mail: satoshin@kyumed.jp

Key words: cardiac index, chronic heart failure, exercise training, exercise tolerance, vascular resistance

Received: February 05, 2015; Accepted: March 10, 2015; Published: March 14 2015 


\section{Statistical analysis}

Because the continuous parameters in the present study were found to exhibit a non-normal distribution, the data are expressed as the medians (interquartile range) for continuous variables and as numbers and percentages for categorical variables. We therefore performed the statistical analysis using the nonparametric method. Comparisons of continuous variables between two groups were made using the MannWhitney test or Wilcoxon signed-rank test. The chi-square test or Fisher's exact test was used to compare categorical variables. A twotailed $p$-value $<0.05$ was considered to be statistically significant. All statistical analyses were performed using the GraphPad Prism ${ }^{\circledR} 5.0$ (GraphPad Software, Inc., San Diego, USA) software package.

\section{Results}

The baseline demographic and clinical characteristics of the patients are summarized in Table 1. There were no significant differences in the gender distribution. The basic diseases underlying the heart failure were idiopathic dilated cardiomyopathy in 22 patients, valvular heart disease in 22 , old myocardial infarction in 15 , congenital heart disease in two and hypertensive heart disease in one.

A comparison of the cardiac parameters is summarized in Table 2. There were no significant differences in the mean blood pressure before and after exercise (Figure 1), whereas the heart rate was significantly increased after exercise (Figure 2). While the total peripheral vascular resistance index tended to be decreased after exercise (Figure 4), the cardiac index tended to be increased (Figure 5).

Table 1. The baseline demographic and clinical characteristics of the patients.

\begin{tabular}{|l|c|c|}
\hline & & p value \\
\hline Age (years) & $63(49-72)$ & \\
\hline Left ventricular ejection fraction (\%) & $62(39-69)$ & \\
\hline Sex, Male (\%) & $39(62.9)$ & 0.0068 \\
\hline
\end{tabular}

The data are expressed as the median (interquartile range) for continuous variables

Table 2. A comparison of the hemodynamic parameters before and after exercise.

\begin{tabular}{|l|l|l|l|}
\hline & Pre-Ex & Post-Ex & p value \\
\hline Mean blood pressure $(\mathrm{mmHg})$ & $89(77-100)$ & $89(80-99)$ & 0.8501 \\
\hline Heart rate $(\mathrm{bpm})$ & $74(65-85)$ & $76(67-89)$ & 0.002 \\
\hline $\begin{array}{l}\text { Total peripheral vascular resistance } \\
\left.\text { index (dynes sec/ } \mathrm{cm}^{5} \mathrm{~m}^{2}\right)\end{array}$ & $2459(1883-3239)$ & $2192(1771-3148)$ & 0.1061 \\
\hline Cardiac index $\left(\mathrm{L} / \mathrm{min}^{2} \mathrm{~m}^{2}\right)$ & $3.1(2.5-3.7)$ & $3.3(2.5-4.2)$ & 0.1637 \\
\hline
\end{tabular}

The data are expressed as the median (interquartile range) for continuous variables

\section{$H R$, rest vs Ex}

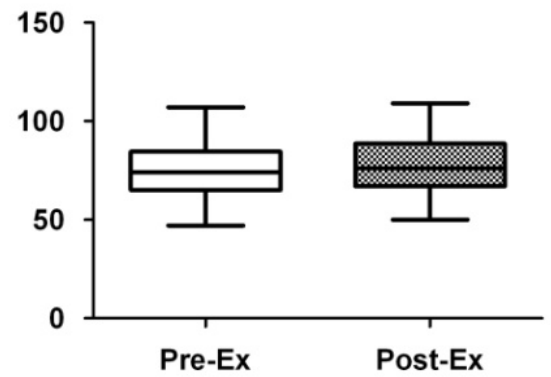

Figure 1. Comparison of heart rate before and after exercise. Box plots indicate medians, $25^{\text {th }}$ and $75^{\text {th }}$ percentiles (boxes), and minimum and maximum values (whiskers). Pre-Ex: before exercise; Post-Ex: after exercise.

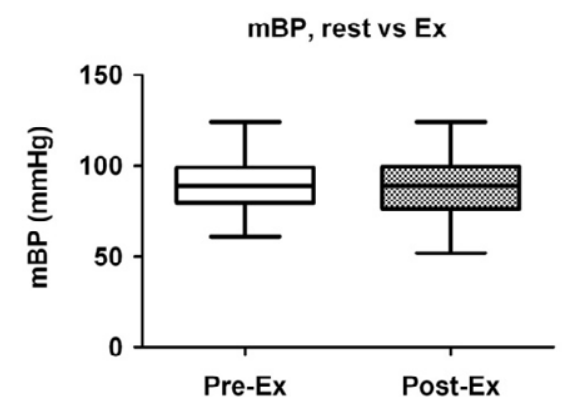

Figure 2. Comparison of mean blood pressure before and after exercise. Box plots indicate medians, $25^{\text {th }}$ and $75^{\text {th }}$ percentiles (boxes), and minimum and maximum values (whiskers). Pre-Ex: before exercise; Post-Ex: after exercise.

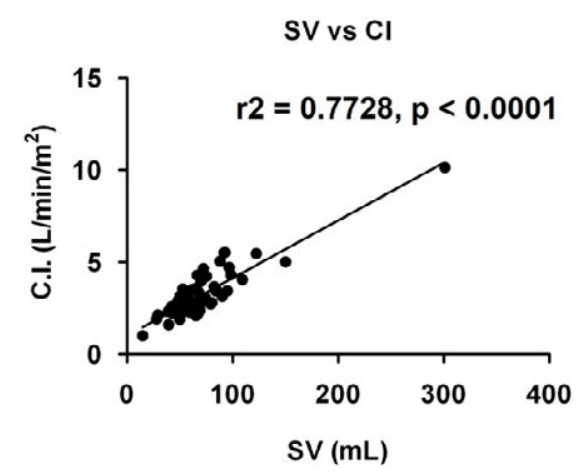

Figure 3. Scatterplots showing correlations between stroke volume (SV) and cardiac index (C.I.). $\mathrm{r}^{2}$ : correlation efficient.

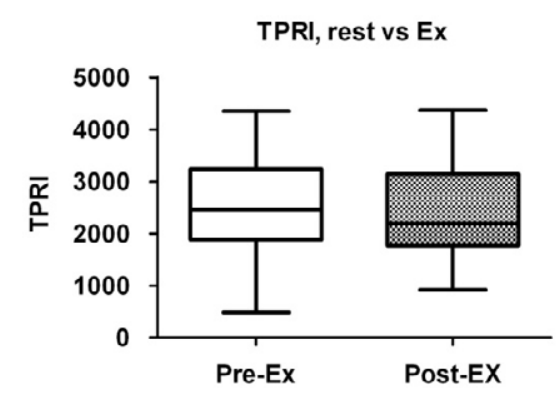

Figure 4. Comparison of total peripheral resistance index (TPRI) before and after exercise Box plots indicate medians, $25^{\text {th }}$ and $75^{\text {th }}$ percentiles (boxes), and minimum and maximum values (whiskers). Pre-Ex: before exercise; Post-Ex: after exercise.

\section{Discussion}

The present study demonstrated a possibility that in stable patients with chronic heart failure being treated with oral medications, exercise training affects the vascular resistance to decrease the total peripheral vascular resistance, which may thus lead to increased cardiac output. This may be one of the beneficial effects of exercise training in patients with chronic heart failure.

A previous study demonstrated that exercise training improves the endothelial function via the restoration of the nitric oxide production [5], thus improving the aerobic capacity [3], delaying the anaerobic metabolism [2], and restoring the autonomic balance, which was 


\section{$\mathrm{Cl}$, rest vs Ex}

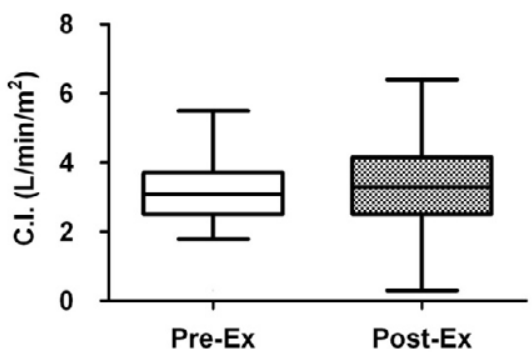

Figure 5. Comparison of cardiac index (C.I.) before and after exercise. Box plots indicate medians, $25^{\text {th }}$ and $75^{\text {th }}$ percentiles (boxes), and minimum and maximum values (whiskers). Pre-Ex: before exercise; Post-Ex: after exercise.

associated with lower rates of mortality [3] and hospital readmission for heart failure during a long-term follow-up [6].

However, the physiological mechanisms underlying the preserved exercise tolerance in patients with stable chronic heart failure [7], irrespective of the decreased ejection fraction and acute effects of exercise in these patients, have been unclear. Decreased peripheral vascular resistance could lead to an increase in stroke volume, thus could be associated with an increase in cardiac output because cardiac index is strongly correlated with stroke volume (Figure 3 ). We suggest that our findings demonstrate the possible underlying mechanism.

The following study limitations should be noted. This study was a small non-randomized observational study encompassing a selection bias. This may be the reason why the changes in these parameters did not reach statistical significance.

In conclusion, exercise training affects the vascular resistance to decrease the total peripheral vascular resistance, and thus may lead to increased cardiac output. Exercise-induced peripheral vasodilation may underlie. This may be one of beneficial effects of exercise training in patients with chronic heart failure.

\section{Acknowledgement}

The authors are very grateful to the technical staff in the Departments of Clinical Laboratory and Radiology at the National Hospital Organization Kyushu Medical Center for their expert technical assistance. We have no conflicts of interest to declare in association with this study.

\section{References}

1. McKelvie RS, Teo KK, McCartney N, Humen D, Montague T, et al. (1995) Effects of Exercise Training in Patients With Congestive Heart Failure: A Critical Review. J Am Coll Cardiol 25: 789-796. [Crossref]

2. Gielen S, Schuler G, Adams V (2010) Cardiovascular Effects of Exercise Training Molecular Mechanisms. Circulation 122: 1221-1238. [Crossref]

3. Gokce N, Vita A, Bader DS, Sherman DL, Hunter LM, et al. (2002) Effect of Exercise on Upper and Lower Extremity Endothelial Function in Patients with Coronary Artery Disease. Am J Cardiol 90: 124-127. [Crossref]

4. Belardinelli R, Georgiou D, Cianci G, Purcaro A (2012) 10-Year Exercise Training in Chronic Heart Failure. J Am Coll Cardiol 60: 1521-1528. [Crossref]

5. Linke A, Schoene N, Gielen S, Hofer J, Erb S, et al. (2001) Endothelial Dysfunction in Patients with Chronic Heart Failure: systemic effects of lower-limb exercise training. $J$ Am Coll Cardiol 37: 392-397. [Crossref]

6. Hambrecht R, Wolff A, Gielen S, Linke A, Hofer J, et al. (2000) Effects of Exercise on Coronary Endothelial Function in Patients with Coronary Artery Disease. $N$ Eng $J$ Med 342: 454-460. [Crossref]

7. Clark AL, Poole-Wilson PA, Coats AJS (1996) Exercise Limitation in Chronic Heart Failure. J Am Coll Cardiol 28: 1092-1102. [Crossref]

8. Dickstein K (2008) Acute and Chronic Heart Failure. The Task Force on Heart Failure of the European Society of Cardiology (ESC) Developed in collaboration with the Heart Failure Association of the ESC (IHA). Eur Heart J 29: 2388-2342.

9. Anderson JL, Adams CD, Antman EM, Bridges CR, Califf RM, et al. (2007) ACC AHA 2007 guidelines for the management of patients with unstable angina/non-STelevation myocardial infarction. A Report of the American College of Cardiology/ American Heart Association Task Force on Practice Guidelines (Writing Committee to Revise the 2002 Guidelines for the Management of Patients With Unstable Angina/ Non-ST-elevation Myocardial Infarction). Circulation 116: 803-877. [Crossref]

Copyright: (C2015 Satoh S. This is an open-access article distributed under the terms of the Creative Commons Attribution License, which permits unrestricted use, distribution, and reproduction in any medium, provided the original author and source are credited. 